\title{
FERMI GAS WITH 4-FERMION BCS-TYPE INTERACTION
}

\author{
J. MaĆKowiak and P. TARASEWICZ \\ Institute of Physics, N. Copernicus University \\ Grudziądzka 5/7, 87-100 Torun, Poland
}

(Received September 2, 1997)

\begin{abstract}
A gas of spin $1 / 2$ fermions with an interaction $V+W=$ $-\sum_{k, k^{\prime}} g_{k k^{\prime}} b_{k}^{*} b_{-k}^{*} b_{k^{\prime}} b_{-k^{\prime}}+\sum_{k} \gamma_{k} b_{k}^{*} b_{k}$, where $b_{k}=a_{k+} a_{k-}$ and $a_{k \sigma}, a_{\boldsymbol{k}^{\prime} \sigma^{\prime}}^{*}$ satisfy Fermi anticommutation relations, is investigated. The trial ground state $|G\rangle$ is similar in form to the BCS ground state, with $b_{k}^{*} b_{-k}^{*}$ replacing $a_{k+}^{*} a_{-k-}^{*}$, but because the excitation energies are not simply additive, the trial density matrix $\rho_{0}$ differs from the BCS one. The expectation values $\langle G|H| G\rangle$ and $\operatorname{Tr}\left(H \rho_{0}\right)$ are minimized, revealing the presence of a second-order phase transition, with $T_{c}>T_{c(B C S)}$ for appropriately adjusted $\gamma_{k}$. It is shown that the minimization procedure applied leads to an expression for the free energy density of $H$, which is asymptotically exact in the infinite-volume limit. Comparison with experimental data on high-temperature superconductors is made and for a particular choice of $\gamma_{k}$ qualitative agreement is found with the temperature dependence of the order parameter of the BSCCO superconductor.
\end{abstract}

PACS numbers: 74.20.-z, 74.20.Fg

\section{Introduction}

It is generally accepted that current carriers in high- $T_{\mathrm{c}}$ superconductors (HTSC) consist of tightly bound local pairs of spin $1 / 2$ fermions with opposite spins, e.g. [1-4]. Hubbard-type Hamiltonians are usually proposed as theoretical models of HTSC due to the flexibility with which local pairing interactions can be modelled in these systems, e.g. $[1,2,4,5]$.

A system of strongly correlated electrons, set up in the framework of the Hubbard model, the so-called spin liquid, in which the same number of degrees of freedom, corresponding to compensated spin configurations, are removed from either $k$-space or real space, was proposed in Refs. [6-8]. A particular realization of the spin liquid, obtained by adding to the BCS Hamiltonian the term

$$
W=\sum_{k} \gamma_{k} n_{k+} n_{k-1},
$$


where $n_{\boldsymbol{k} \sigma}=a_{\mathbf{k} \sigma}^{*} a_{\mathbb{k} \sigma}$ and $a_{\boldsymbol{k} \sigma}^{*}, a_{\mathbf{k} \sigma}$ are fermion creation and annihilation operators corresponding to momentum $k$ and spin $\sigma$, was investigated in Refs. [9, 10].

The interaction $W$ in the form (1) can be viewed as a pair-binding potential of magnetic origin. However, when written in terms of $b_{\boldsymbol{k}}^{*}=a_{k-}^{*} a_{k+}^{*}, b_{\boldsymbol{k}}=a_{k+} a_{\frac{\pi}{4}-}$, it takes the form

$$
W=\sum_{k} \gamma_{k} b_{k}^{*} b_{k}
$$

reminiscent of the kinetic energy operator of a free quantum gas. In HTSC the quasiparticles represented by the operators $b_{k}^{*}, b_{\boldsymbol{k}}$, as well as fermions which are not bound by $W$, can be expected to interact via the phonon field, owing to the presence of a weak isotope effect in these materials [11-13]. The form of the effective BCS Hamiltonian

$$
H_{\mathrm{BCS}}=T+V_{\mathrm{BCS}}
$$

where

$$
\begin{aligned}
& T=\sum_{k \sigma} \varepsilon_{k} a_{\mathbb{k} \sigma}^{*} a_{k \sigma}, \quad \varepsilon_{k}=k^{2} / 2 m-\mu, \\
& V_{\mathrm{BCS}}=-\sum_{\mathbb{k}, \mathbb{k}^{\prime}} G_{\boldsymbol{k} \mathbb{k}^{\prime}} a_{k+}^{*} a_{-k_{-}-}^{*} a_{-\mathbb{k}^{\prime}-} a_{k^{\prime}}+
\end{aligned}
$$

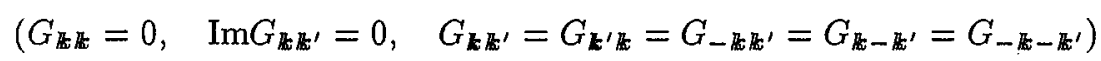

and the similarity between $W$ and $T$ suggests therefore a 4 -fermion operator of the form

$$
V=-\sum_{k, \mathbb{k}^{\prime}} g_{\mathbf{k} \mathbb{k}^{\prime}} b_{k}^{*} b_{-\mathbb{k}^{\prime}}^{*} b_{-\mathbf{k}^{\prime}} b_{k^{\prime}}
$$

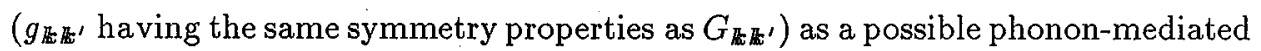
attraction between bound pairs in a HTSC and a full Hamiltonian of the form

$$
H^{\prime}=H_{\mathrm{BCS}}+W+V \text {. }
$$
and

In the present paper we deal with a simplified version of $H^{\prime}$ in which $V_{\mathrm{BCS}}=0$

$$
H=T+W+V .
$$

The functions $\gamma_{k}, g_{\boldsymbol{k}^{\prime} \mathbf{m}^{\prime}}$ in $H$ will remain unspecified, the only assumption being that their particular form should be adjusted so as to obtain the best possible agreement of the resulting theory with experiment.

In Secs. 2-4 minimization procedures for the ground-state energy of $H$ and free energy of $H$ are performed. The trial ground state $|G\rangle$ for $H$ is similar in form to the BCS ground state [14], but the quasiparticle excitation energies from $|G\rangle$ are not simply additive. The structure of excited states and excitation spectrum define the structure of the trial grand canonical density matrix $\rho_{0}$ and minimization of the free energy $\mathcal{F}\left[\rho_{0}\right]$ determines $\rho_{0}$ uniquely. The order parameter $\Delta_{\boldsymbol{k}}$ satisfies a gap equation which has a non-zero soluticn below $T_{c}$, proving that $T_{\mathrm{c}}$ is the temperature at which the system undergoes a 2 nd order phase transition. At $T>T_{\mathrm{c}}$ the system behaves like a gas of free fermions and free bound pairs, whereas 
below $T_{\mathrm{c}}$ the interaction $V$ takes full effect and the interacting Fermi gas with the Hamiltonian $H$ behaves like a gas of fermion quasiparticles with energies which, in general, are not additive.

If $\gamma_{k}=0$, then $T_{c}<T_{\mathrm{c}(\mathrm{BCS})}$ for the same values of $g_{k k^{\prime}}$, conduction half-bandwidth $\delta$ and density of states $\rho$ near the Fermi level $\mu_{\mathrm{F}}=k_{\mathrm{F}}^{2} / 2 \mathrm{~m}$ as in the one-parameter BCS model [14]. The interaction $V$ is thus weaker than $V_{\mathrm{BCS}}$, in agreement with weakness of the isotope effect in HTSC. However, $T_{\mathrm{c}}>T_{\mathrm{c}(\mathrm{BCS})}$ if $\left|\varepsilon_{k}+\gamma_{k} / 2\right|$ is sufficiently small, suggesting that $H$ could, possibly, serve as a model of HTSC.

In Sec. 5 a test on validity of this conjecture is performed by comparing experimental data on $\Delta(\beta)$ for the BSCCO superconductor with theoretical predictions for the choice $\gamma\left(\varepsilon_{k}\right)=-2\left|\varepsilon_{k}\right|$ in $W$. Qualitative agreement is found between predictions of the model and experiment.

In Sec. 6 it is shown by a method of Czerwonko [10] that the minimization procedures applied to $H$ in Secs. 2-4 yield an expression for the free energy density which is asymptotically exact in the infinite-volume limit.

\section{The ground state}

Since $V$ and $V_{\mathrm{BCS}}$ are similar in form, the appropriate trial ground state $|G\rangle$ for $H$ can be expected to be similar to the BCS ground state $|\mathrm{BCS}\rangle$, with the

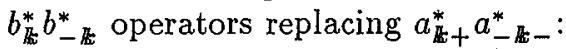

$$
|G\rangle=\prod_{k>0}\left(u_{k}+v_{k_{k}} b_{k}^{*} b_{-k}^{*}\right)|0\rangle,
$$

where $k: k>0\}$ stands for the set of vectors with one fixed, but arbitrary, component positive and $u_{k}, v_{k}$ are real variational parameters satisfying $u_{k}^{2}+v_{k}^{2}=1$. Obviously, $u_{k}=u_{-k}, v_{k}=v_{-k}$ and $\langle G \mid G\rangle=1$.

For $\langle G|H| G\rangle$ one obtains

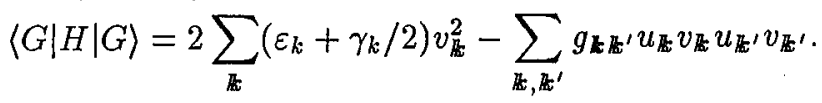

This expression is similar to the expectation value

$$
\left\langle\mathrm{BCS}\left|H_{\mathrm{BCS}}\right| \mathrm{BCS}\right\rangle=\left\langle\mathrm{BCS}\left|T+V_{\mathrm{BCS}}\right| \mathrm{BCS}\right\rangle \text {. }
$$

The only difference is that $\varepsilon_{k}+\gamma_{k} / 2$ in (7) replaces $\varepsilon_{k}$ in $\left\langle\mathrm{BCS}\left|H_{\mathrm{BCS}}\right| \mathrm{BCS}\right\rangle$. The $u_{k}, v_{k}$ which minimize $\langle G|H| G\rangle$ are therefore analogous to the BCS ones:

$$
u_{\mathrm{k}}^{2}=\frac{1}{2}\left(1+\nu_{k} E_{\boldsymbol{k}}^{-1}\right), \quad v_{k}^{2}=\frac{1}{2}\left(1-\nu_{k} E_{\boldsymbol{k}}^{-1}\right),
$$

where $\nu_{k}=\varepsilon_{k}+\gamma_{k} / 2, E_{k}=\left(\nu_{k}^{2}+\Delta_{k}^{2}\right)^{1 / 2}$ and $\Delta_{k}=\sum_{k^{\prime}} g_{k} k^{\prime} u_{\mathbb{k}^{\prime}} v_{k^{\prime}}$ is the solution of the zero-temperature gap equation

$$
\Delta_{\mathbb{R}^{2}}=\frac{1}{2} \sum_{\mathbf{k}^{\prime}} g_{\boldsymbol{k}^{\prime} \boldsymbol{k}^{\prime}} \Delta_{\boldsymbol{k}^{\prime}} E_{\boldsymbol{k}^{\prime}}^{-1} .
$$

For $g_{k^{\prime} k^{\prime}}=g \chi(k) \chi\left(k^{\prime}\right)$ if $k \neq k^{\prime}(\chi(k)$ denoting the characteristic function of the set $\left.\left\{k: \varepsilon_{k} \in[\mu-\delta, \mu+\delta]\right\}\right)$ and $\gamma_{k}=2 \gamma \varepsilon_{k}, \gamma>-1$, the equation for $\mu$, viz.,

$$
\left\langle G\left|\sum_{k \sigma} a_{\text {范 } \sigma}^{*} a_{k \sigma}\right| G\right\rangle=n,
$$


where $n$ denotes the number of fermions, has a solution $\mu \neq \mu_{\mathrm{F}}$ and the nontrivial solution of Eq. (9) is $\Delta_{k}=\Delta \chi(k)$, with

$$
\begin{aligned}
& \Delta=2 \sinh \left[\frac{2(1+\gamma)}{g \rho}\right] \\
& \times\left\{(\mu \gamma)^{2}+\delta^{2}(1+\gamma)^{2}-\left[(\mu \gamma)^{2}-\delta^{2}(1+\gamma)^{2}\right] \cosh \left[\frac{2(1+\gamma)}{g \rho}\right]\right\}^{1 / 2} .
\end{aligned}
$$

If $\gamma_{k}=0$, then $\mu=\mu_{\mathrm{F}}$ and $\Delta_{k}=\delta\left[\sinh (g \rho)^{-1}\right]^{-1} \chi(k)$. The proofs are given in Appendix A.

Similarly as in the BCS model,

$$
E_{G}=\langle G|H| G\rangle=\sum_{k}\left[\nu_{k}\left(1-\nu_{k} E_{\mathbb{k}}^{-1}\right)-\frac{1}{2} \Delta_{k}^{2} E_{k}^{-1}\right]
$$

suggesting that the ground-state properties of the system with the Hamiltonian $H$ are analogous to those of $H_{\mathrm{BCS}}$ except for the shift of the Fermi level $\mu$. This conjecture is proved in Sec. 6 by investigating the infinite-volume limit for $H$. The 3-dimensional Hubbard model in the weak-coupling limit or, equivalently, at low temperatures $[4,15,16]$ and some 2-dimensional local systems [17] also exhibit BCS-type behaviour.

\section{Excited states}

Having found the best ground state vector $|G\rangle$ in the set of trial vectors introduced in Sec. 2, the approximate excited states can be determined by proceeding similarly as Bogolyubov and Valatin with $|\mathrm{BCS}\rangle[18,19]$, i.e. by first solving the equation for $\alpha$

$$
\alpha|G\rangle=0 \text {. }
$$

The solutions of this equation can be most convenie:atly written using the notation $a_{\mathbb{k} 1}:=a_{k+}, a_{\mathbb{k} 2}:=a_{\mathbb{k}-}, a_{\mathbb{k} 3}:=a_{-k+}, a_{\mathbb{k} 4}:=a_{-k-}$. Equation (12) is then satisfied by

$$
\begin{array}{ll}
\alpha_{k 1}=u_{k} a_{k 1}-v_{k} a_{k 2}^{*} a_{k 3}^{*} a_{k 4}^{*}, & \alpha_{k 2}=u_{k} a_{k 2}+v_{k} a_{k 3}^{*} a_{k 4}^{*} a_{k 1}^{*}, \\
\alpha_{k 3}=u_{k} a_{k 3}-v_{k} a_{k 1}^{*} a_{k 2}^{*} a_{k 4}^{*}, & \alpha_{k 4}=u_{k} a_{k 4}+v_{k} a_{k 1}^{*} a_{k 2}^{*} a_{k 3}^{*} .
\end{array}
$$

The operators $\alpha_{\boldsymbol{k} i}$ are anticommuting

$$
\left[\alpha_{\mathbf{k}^{\prime}}, \alpha_{\mathbf{k}^{\prime} j}\right]_{+}=\alpha_{\mathbf{k} i} \alpha_{\mathbf{k}^{\prime} j}+\alpha_{\mathbf{k}^{\prime} j} \alpha_{k i}=0,
$$

but

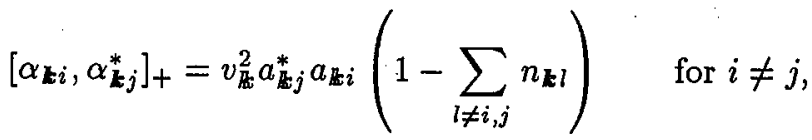

$$
\begin{aligned}
& {\left[\alpha_{\boldsymbol{k} i}, \alpha_{\boldsymbol{k} i}^{*}\right]_{+}=1+v_{\boldsymbol{k}}^{2}\left(\sum_{i \neq j<l \neq i} n_{\boldsymbol{k} j} n_{\boldsymbol{k} l}-\sum_{j \neq i} n_{\mathbf{k} j}\right)}
\end{aligned}
$$

where $n_{k i}=a_{k i}^{*} a_{\mathbb{E} i}$. 
The normalized $k$-excited states are therefore (cf. $[18,19])$ represented by the following vectors:

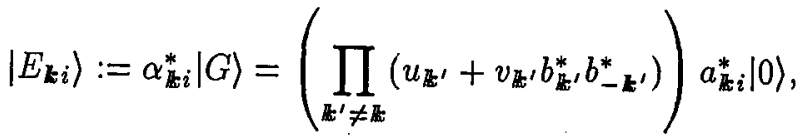

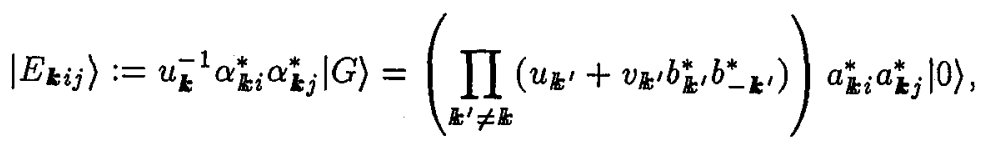

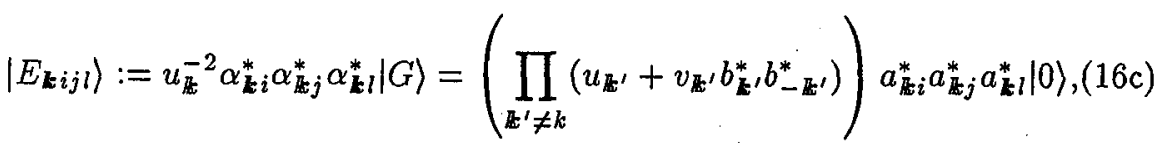

$$
\begin{aligned}
& \left|E_{\mathbf{k} 1234}\right\rangle:=u_{\mathbf{k}}^{-2} \alpha_{\mathbf{k} 1}^{*} \alpha_{\mathbf{k} 2}^{*} \alpha_{\mathbb{k} 3}^{*} \alpha_{\mathbf{k} 4}^{*}|G\rangle \\
& =\left(\prod_{\boldsymbol{k}^{\prime} \neq \mathbb{k}}\left(u_{\boldsymbol{k}^{\prime}}+v_{\mathbb{k}^{\prime}} b_{\mathbb{k}^{\prime}}^{*}, b_{-\mathbb{k}^{\prime}}^{*}\right)\right)\left(u_{\boldsymbol{k}} b_{k}^{*} b_{-\mathbb{k}}^{*}-v_{\mathbb{k}}\right)|0\rangle .
\end{aligned}
$$

According to Eqs. (13)-(15), the excitations represented by the operators $\alpha_{\boldsymbol{k} i}^{*}$ are neither fermions nor bosons. Nonetheless, some of their properties are the same as those of particles obeying Fermi or Bose statistics: the set of vectors (16a-d), with varying $k$, is an orthonormal system, the number of excitations in any state (16a-d) can be lowered by acting on it with the operators $\alpha_{\mathbb{k} i}$, e.g.,

$$
\begin{aligned}
& \alpha_{k i k}\left|E_{\boldsymbol{k} i j}\right\rangle=u_{k}\left|E_{\boldsymbol{k}_{j}}\right\rangle \quad \text { for } i \neq j, \\
& \alpha_{\boldsymbol{k} i}\left|E_{\mathbf{k} j l}\right\rangle=0 \quad \text { for } i \neq j, i \neq l .
\end{aligned}
$$

The vectors $(16 \mathrm{a}-\mathrm{d})$ are eigenvectors of the operators $\alpha_{\mathbf{k} i}^{*} \alpha_{\boldsymbol{k} i}$, viz.,

$$
\begin{aligned}
& \alpha_{\mathbf{k} i}^{*} \alpha_{\mathbf{k} i}\left|E_{\mathbf{k} i}\right\rangle=\left|E_{\mathbf{k} i}\right\rangle, \\
& \alpha_{\boldsymbol{k} i}^{*} \alpha_{\boldsymbol{k} i}\left|E_{\mathbf{k} i j}\right\rangle=u_{\boldsymbol{k}}^{2}\left|E_{\mathbf{k} i j}\right\rangle, \\
& \alpha_{k i}^{*} \alpha_{\mathbf{k} i}\left|E_{\mathbf{k} i j l}\right\rangle=u_{k i}^{2}\left|E_{\boldsymbol{k} i j l}\right\rangle, \\
& \alpha_{k i}^{*} \alpha_{\mathbf{k} i}\left|E_{\mathbf{k} 1234}\right\rangle=\left|E_{\mathbf{k} 1234}\right\rangle,
\end{aligned}
$$

but, as implied by these equations, in general, their eigenvalues are not equal to the number of excitations present in the eigenvector. The excitation energies from the ground state $|G\rangle$ are equal

$$
\begin{aligned}
& \left\langle E_{\mathbb{k} i}|H| E_{\boldsymbol{k} i}\right\rangle-E_{G}=2 E_{\mathbf{k}}-\varepsilon_{k}-\gamma_{k}, \\
& \left\langle E_{k i j}|H| E_{\mathbf{k} i j}\right\rangle-E_{G}=2 E_{k}-\gamma_{k}, \text { if }(i, j) \in\{(1,3),(1,4),(2,3),(2,4)\},(10) \\
& \left\langle E_{\mathbb{k} i j}|H| E_{\mathbf{k} i j}\right\rangle-E_{G}=2 E_{\mathbf{k}} \text {, if }(i, j) \in\{(1,2),(3,4)\}, \\
& \left\langle E_{k i j l}|H| E_{k i j l}\right\rangle-E_{G}=2 E_{\boldsymbol{k}}+\varepsilon_{k}, \quad i<j<l, \\
& \left\langle E_{\mathbb{1 2} 1234}|H| E_{\mathbf{k} 1234}\right\rangle-E_{G}=4 E_{\mathbf{k}} \text {. }
\end{aligned}
$$


It follows that, unlike in BCS theory, these energies are not simply additive when counted in the $k$-space spanned by $\left|G_{k}\right\rangle=\left(u_{k}+v_{\mathbb{k}} b_{k}^{*} b_{-\mathbb{k}}^{*}\right)|0\rangle$ and the vectors $(16 \mathrm{a}-\mathrm{d})$.

The structure of the subspace $M_{k}=M_{-k}$ spanned by the orthonormal basis $B_{\mathbb{k}}$ consisting of $\left|G_{\boldsymbol{k}}\right\rangle$ and vectors (16a-d), shows that there also exist fermion operators $c_{\boldsymbol{k} \sigma}$ which annihilate the vector $\left|G_{\boldsymbol{k}}\right\rangle$. To construct these fermion operators let us introduce the unitary transformation $U_{k}=U_{-k}$ defined as

$$
\begin{aligned}
U_{k} & :=\sum_{i}\left|E_{\mathbf{k} i}\right\rangle\left\langle E_{k \mathbf{k} i}\left|+\sum_{i<j}\right| E_{\mathbf{k} i j}\right\rangle\left\langle E_{k i j}\left|+\sum_{i<j<l}\right| E_{\mathbf{k} i j l}\right\rangle\left\langle E_{\mathbb{k} i j l}\right| \\
& +\left|G_{\boldsymbol{k}}\right\rangle\left\langle 0|+| E_{\mathbf{k} 1234}\right\rangle\langle 0| a_{\mathbb{k} 4} a_{\mathbb{k} 3} a_{\mathbb{k} 2} a_{\mathbf{k} 1} .
\end{aligned}
$$

Obviously, $U_{k}|0\rangle=\left|G_{\boldsymbol{k}}\right\rangle$ and

$$
\begin{aligned}
& \left.0=U_{\mathbb{k}} a_{k \sigma}|0\rangle=U_{k_{k}} a_{\mathbb{k} \sigma} U_{k}^{*} U_{k_{k}}|0\rangle=: c_{k} \sigma G_{\boldsymbol{k}}\right\rangle, \\
& 0=U_{k} a_{-\pi \sigma}|0\rangle=U_{\mathbb{k}} a_{-\pi \sigma} U_{k}^{*} U_{k}|0\rangle=: c_{-\pi \sigma}\left|G_{k}\right\rangle .
\end{aligned}
$$

Since $c_{\mathbb{k} \sigma}$ results by transforming unitarily $a_{\mathbb{k} \sigma}$, the anticommutation relations between the operators $a_{\boldsymbol{k} \sigma}, a_{\boldsymbol{k}_{\sigma}}^{*}, a_{-\boldsymbol{k} \sigma}, a_{-\boldsymbol{k} \sigma}^{*}$, are preserved by $c_{\boldsymbol{k} \sigma}, c_{\boldsymbol{k}_{\sigma}}^{*}, c_{-\boldsymbol{k} \sigma}, c_{-\mathbf{k} \sigma}^{*}$. The structure of excited states $(16 \mathrm{a}-\mathrm{d})$ is also preserved, since in $M_{\mathbb{k}}$, e.g.,

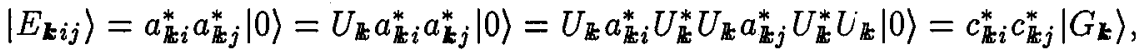

$$
\begin{aligned}
& \left|E_{\boldsymbol{k} 1234}\right\rangle=U_{\mathbb{k}} a_{\mathbb{k} 1}^{*} a_{\mathbb{k} 2}^{*} a_{\mathbb{k} 3}^{*} a_{\mathbf{k} 4}^{*}|0\rangle=c_{\mathbb{k} 1}^{*} c_{k 2}^{*} c_{k 3}^{*} c_{k 4}^{*}|0\rangle .
\end{aligned}
$$

\section{Minimization of the free energy}

The form of the trial grand canonical density matrix $\rho_{0}$ is determined by the structure of $|G\rangle$, the excited states (16a-d) and the excitation energies (19a-e). In order to write down $\rho_{0}$, let us define the projectors

$$
\begin{aligned}
& P_{\mathbb{k} 0}=\left|G_{\boldsymbol{k}}\right\rangle\left\langle G_{\mathbb{k}}\left|, \quad P_{\mathbb{k} i}=\right| E_{\mathbf{k} i}\right\rangle\left\langle E_{\mathbb{k} i}\left|, \quad P_{\mathbb{k} i j}=\right| E_{\boldsymbol{k} i j}\right\rangle\left\langle E_{\mathbb{k} i j}\right|, \\
& P_{\mathbb{k} i j l}=\left|E_{\mathbf{k} i j l}\right\rangle\left\langle E_{\boldsymbol{k} i j l}\left|, \quad P_{\mathbb{k} 1234}=\right| E_{\mathbf{k} 1234}\right\rangle\left\langle E_{\mathbb{k} 1234}\right| .
\end{aligned}
$$

Since there exist two types of two-quasiparticle excitation energies, whereas the excitation energies of one- and three-quasiparticle excitations do not depend on the type of quasiparticles involved, therefore

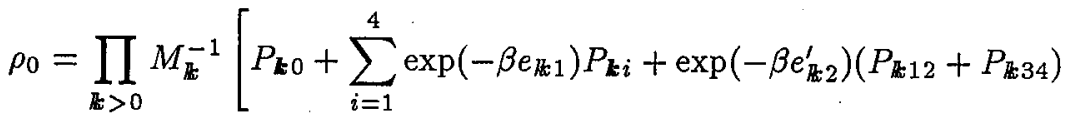

$$
\begin{aligned}
& +\exp \left(-\beta e_{k 2}\right)\left(P_{k 13}+P_{\mathbb{k} 14}+P_{k 23}+P_{\mathbf{k} 24}\right) \\
& \left.+\sum_{i<j<l} \exp \left(-\beta e_{R_{k} 3}\right) P_{\mathbf{k} i j l}+\exp \left(-\beta e_{\mathbb{k} 4}\right) P_{\mathbb{k 1 2 3 4}}\right] \text {, }
\end{aligned}
$$

where

$$
M_{k}=1+\sum_{i=1}^{3} 4 \exp \left(-\beta e_{k i}\right)+2 \exp \left(-\beta e_{k t 2}^{\prime}\right)+\exp \left(-\beta e_{k 4}\right) .
$$


In terms of

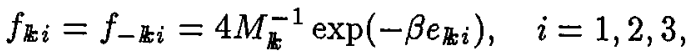

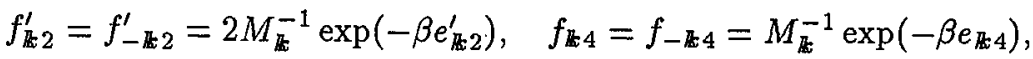

$\rho_{0}$ takes the form

$$
\begin{aligned}
& \rho_{0}=\prod_{k>0}\left[\left(1-\sum_{i=1}^{4} f_{k i}-f_{k 2}^{\prime}\right) P_{k 0}+\frac{1}{4} \sum_{i=1}^{4} f_{k 1} P_{\mathbf{k} i}+\frac{1}{2} f_{k 2}^{\prime}\left(P_{k 12}+P_{k 34}\right)\right. \\
& \left.+\frac{1}{4} f_{k 2}\left(P_{k 13}+P_{k 14}+P_{k 23}+P_{k 24}\right)+\frac{1}{4} \sum_{i<j<l} f_{k 3} P_{k i j l}+f_{k 4} P_{k-1234}\right] .
\end{aligned}
$$

The entropy $\mathcal{S}\left[\rho_{0}\right]$ equals

$$
\begin{aligned}
& \mathcal{S}\left[\rho_{0}\right]=-(T \beta)^{-1} \operatorname{Tr}\left(\rho_{0} \ln \rho_{0}\right) \\
&=\frac{1}{2}(T \beta)^{-1} \sum_{k}\left[\left(1-\sum_{i=1}^{4} f_{k i}-f_{k 2}^{\prime}\right) \ln \left(1-\sum_{j=1}^{4} f_{k j}-f_{k 22}^{\prime}\right)\right. \\
&\left.+f_{k 1} \ln \frac{1}{4} f_{k 1}+f_{\mathbb{k} 2} \ln \frac{1}{4} f_{k 2}+f_{k 2}^{\prime} \ln \frac{1}{2} f_{k 22}^{\prime}+f_{k 3} \ln \frac{1}{4} f_{k 3}+f_{k 4} \ln f_{k 44}\right]
\end{aligned}
$$

and the average energy in the state $\rho_{0}$

$$
\begin{aligned}
& \mathcal{E}\left[\rho_{0}\right]=\operatorname{Tr}\left(H \rho_{0}\right)=2 \sum_{k} \nu_{k}\left[\left(1-\sum_{i=1}^{4} f_{k i}-f_{k 4}-f_{k 2}^{\prime}\right) v_{k}^{2}+\frac{1}{4} \sum_{j=1}^{4} j f_{k j}\right. \\
& \left.+\frac{1}{2} f_{k 2}^{\prime}\right]-\sum_{\mathbb{k}^{\prime}} \gamma_{k^{\prime}}\left(\frac{1}{4} f_{k^{\prime} 1}+\frac{1}{2} f_{k^{\prime} 2}+\frac{1}{4} f_{k^{\prime} 3}\right)-\sum_{k, k^{\prime}} g_{\mathbb{R}^{\prime} k^{\prime}} u_{\mathbb{k}} v_{\mathbb{k}^{\prime}} u_{\mathbf{k}^{\prime}} v_{k^{\prime}} \\
& \times\left(1-\sum_{i=1}^{4} f_{k i}-f_{k 4}-f_{k 2}^{\prime}\right)\left(1-\sum_{j=1}^{4} f_{k^{\prime} j}-f_{k k^{\prime} 4}-f_{k / 2}^{\prime}\right) .
\end{aligned}
$$

The free energy $\mathcal{F}\left[\rho_{0}\right]=\mathcal{E}\left[\rho_{0}\right]-T \mathcal{S}\left[\rho_{0}\right]$ is minimized by the appropriate solutions of the equations

$$
\frac{\partial \mathcal{F}}{\partial f_{k k 2}^{\prime}}=0, \quad \frac{\partial \mathcal{F}}{\partial f_{k i}}=0, \quad i=1,2,3,4
$$

and

$$
\frac{\partial \mathcal{F}}{\partial v_{\text {R }}}=0
$$

The unique solutions of Eqs. (24), expressed in terms of

$$
\Delta_{k}=\sum_{k^{\prime}} g_{k \mathbb{k}^{\prime}} u_{\mathbb{k}^{\prime}} v_{\mathbb{k}^{\prime}}\left(1-\sum_{j=1}^{4} f_{k^{\prime} j}-f_{k^{\prime} 4}-f_{k^{\prime} 2}^{\prime}\right)
$$


and $E_{k}=\left(\nu_{k}^{2}+\Delta_{k}^{2}\right)^{1 / 2}$, are

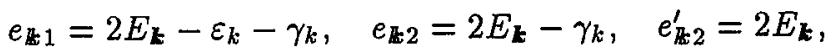

$$
\begin{aligned}
& e_{\mathbb{k} 3}=2 E_{k}+\varepsilon_{k}, \quad e_{k 4}=4 E_{k} .
\end{aligned}
$$

These excitation energies have the same form as those previously found in $(19 \mathrm{a}-\mathrm{d})$.

It follows from (27) that the gap $\Gamma(\beta)$ in the excitation spectrum depends on the form of $\gamma_{k}$ and is present already at $T>T_{\mathrm{c}}$. In this range of temperatures

$$
\Gamma(\beta)=\inf _{k_{k}}\left(2\left|\varepsilon_{k}+\gamma_{k} / 2\right|-\varepsilon_{k}-\gamma_{k}\right) \text {. }
$$

At $T<T_{\mathrm{c}}$ the gap $\Gamma(\beta)$ widens with increasing $\Delta(\beta)$ and equals, according to $(27)$,

$$
\Gamma(\beta)=\inf _{k}\left(2 \sqrt{\left(\varepsilon_{k}+\gamma_{k} / 2\right)^{2}+\Delta_{k}^{2}}-\varepsilon_{k}-\gamma_{k}\right) .
$$

The presence of a gap above and below $T_{c}$ in HTSC has been observed experimentally (cf. e.g. [4]).

As for the density matrix $\rho_{0}$, with $e_{k i}, e_{k 2}^{\prime}$ given by (27), it can be written in the standard form

$$
\rho_{0}=\left[\operatorname{Tr} \exp \left(-\beta h_{0}\right)\right]^{-1} \exp \left(-\beta h_{0}\right),
$$

but $h_{0}$ is not expressible as $E_{G}+\sum_{\mathbb{R} j} h_{\mathbb{k} j} \alpha_{\boldsymbol{k} j}^{*} \alpha_{\mathbb{k} j}$, or $E_{G}+\sum_{\boldsymbol{k} j} h_{\mathbb{k} j} c_{\mathbb{k} j}^{*} c_{\mathbb{k} j}$, as it would be in the case of noninteracting fermions or bosons.

Equation (25) takes the form

$$
2 \nu_{k} u_{\boldsymbol{k}} v_{\boldsymbol{k}}=\Delta_{\boldsymbol{k}}\left(u_{\boldsymbol{k}}^{2}-v_{k}^{2}\right) \text {. }
$$

The solutions $u_{\boldsymbol{k}}, v_{\boldsymbol{k}}$ are the BCS ones given by (8), with $\Delta_{\boldsymbol{k}}$ the solution of the equation

$$
\Delta_{\mathbb{k}}=\frac{1}{2} \sum_{\mathbb{k}^{\prime}} g_{\mathbb{k}_{k^{\prime}}} \frac{\Delta_{\boldsymbol{k}^{\prime}}}{\left(\nu_{k^{\prime}}^{2}+\Delta_{\boldsymbol{k}^{\prime}}^{2}\right)^{1 / 2}} F\left(\beta,\left(\nu_{k^{\prime}}^{2}+\Delta_{\boldsymbol{k}^{\prime}}^{2}\right)^{1 / 2}, \nu_{k^{\prime}}, \gamma_{k^{\prime}}\right)
$$

resulting from (26), where

$$
F(\beta, x, y, z)=\frac{\sinh 2 \beta x}{\cosh 2 \beta x+4 \exp (\beta z / 2) \cosh \beta y+2 \exp \beta z+1} .
$$

Equation (29) is analogous in form to the gap equation of BCS theory, with $F\left(\beta, E_{k}, \nu_{k}, \gamma_{k}\right)$ replacing tanh $\frac{1}{2} \beta E_{\mathbb{k}_{k}}$. The growth and convexity properties of $F(\beta, x, y, z)$ and tanh $\frac{1}{2} \beta x$ are similar:

(A) $F(\beta, x, y, z)$ is vanishing at $x=0$, odd in $x$, increasing in $x$ and concave in $x$ for $x>0$.

(B) For $x>0,2 x>|y|+z / 2>z, F(\beta, x, y, z)$ is increasing in $\beta$.

(C) $\lim F(\beta, x, y, z)=1$ as $\beta \rightarrow \infty$ if $x>0,2 x>|y|+z / 2>z$.

The inequalities in (B), (C) are fulfilled if $\gamma_{k}=-2\left|\varepsilon_{k}\right|+\sigma_{k}$, where $\left|\sigma_{k}\right| \ll\left|\varepsilon_{k}\right|$. It follows therefore that for such $\gamma_{k}$ Eq. (29) is satisfied at all values of $\beta \geq 0$ by the trivial solution $\Delta_{k}=0$ and if $g_{k k^{\prime}} \geq 0$ is nonvanishing on a suitable subset with nonzero Lebesgue measure in $\mathcal{R}^{3} \times \mathcal{R}^{3}$, a non-negative solution $\Delta(\beta, k)$ of this equation exists for values of $\beta$ above some $\beta_{\mathrm{c}} . \Delta(\beta, k)>0$ is increasing in 
$\beta$ in the interval $\left(\beta_{\mathrm{c}}, \infty\right)$ from $\Delta\left(\beta_{\mathrm{c}}, k\right)=0$ to a finite value $\Delta(\infty, k)$, because $x^{-1} F(\beta, x, y, z)$ is decreasing in $x$ for $x>0$. (The proof is given in Appendix B.)

The inequalities in (B), (C) are also satisfied for $\varepsilon_{k}>0$ by $\gamma_{k}=$ $2\left|\varepsilon_{k}\right|+\sigma_{k},\left|\sigma_{k}\right| \ll\left|\varepsilon_{k}\right|$. The growth in $\beta$ of the right hand side of Eq. (29) can be therefore also assured by choosing $\gamma_{k}=2\left|\varepsilon_{k}\right|+\sigma_{k}$ and $g_{k k^{\prime}}=\left[g_{1} \chi_{-}(k)+\right.$ $\left.g_{2} \chi_{+}(k)\right]\left[g_{1} \chi_{-}\left(k^{\prime}\right) g_{2} \chi_{+}\left(k^{\prime}\right)\right]$, where $\chi_{+}(k)=\chi(k) \chi\left[k: \varepsilon_{k}>0\right], \chi_{-}(k)=\chi(k) \chi[k$ : $\left.\varepsilon_{k}<0\right], 0<g_{1} \ll g_{2}$.

The free energy $\mathcal{F}\left[\rho_{0}\right]$ expressed in terms of the solutions of Eqs. (24), (25) takes the form

$$
\begin{aligned}
& \mathcal{F}\left[\rho_{0}\right]=\sum_{\boldsymbol{k}}\left[\nu_{k}-E_{\mathrm{k}}+\left(2 E_{k}\right)^{-1} F\left(\beta, E_{\boldsymbol{k}}, \nu_{k}, \gamma_{k}\right)\left(E_{\mathbf{k}}^{2}-\nu_{k}^{2}\right)\right. \\
& \left.-\frac{1}{2} \beta^{-1} \ln \left(1+\sum_{i=1}^{3} 4 \exp \left(-\beta e_{\mathbb{k} i}\right)+\exp \left(-\beta e_{k 4}\right)+2 \exp \left(-\beta e_{k t 2}^{\prime}\right)\right)\right]
\end{aligned}
$$

and for $\beta \in\left(\beta_{\mathrm{c}}, \infty\right)$ is minimized by the nontrivial solution of Eq. (29). (The proof is given in Appendix C.) As a consequence, there is a second-order phase transition at $\beta_{\mathrm{c}}$.

The equation for $\mu$ at the temperatures $T>0$, viz.,

$$
\operatorname{Tr}\left(\rho_{0} \sum_{k \sigma \sigma} a_{\mathbb{k} \sigma}^{*} a_{\mathbb{k} \sigma}\right)=n
$$

assumes the form

$$
\sum_{k}\left[1-\frac{\nu_{k}}{E_{\mathrm{k}}} F\left(\beta, E_{\mathrm{k}}, \nu_{k}, \gamma_{k}\right)-2 G\left(\beta, E_{\boldsymbol{k}}, \nu_{k}, \gamma_{k}\right)\right]=n
$$

where

$$
G(\beta, x, y, z)=\frac{\exp (\beta z / 2) \sinh (\beta y)}{\cosh (2 \beta x)+4 \exp (\beta z / 2) \cosh (\beta y)+2 \exp (\beta z)+1} .
$$

\section{The critical temperature in the one-parameter model}

In the one-parameter model with $g_{\text {k } k^{\prime}}=g \chi(k) \chi\left(k^{\prime}\right)$ for $k \neq k^{\prime}$ the solution $\Delta_{\mathbb{k}}(\beta)$ of Eq. (29) has the form $\Delta_{\mathbb{E}}(\beta)=\Delta(\beta) \chi(k)$. Equation (29) for the nonzero solution $\Delta(\beta)$ simplifies to

$$
\begin{aligned}
2= & g \rho \int_{-\delta}^{\delta} \frac{\mathrm{d} \varepsilon}{\left\{[\varepsilon+\gamma(\varepsilon) / 2]^{2}+\Delta^{2}\right\}^{1 / 2}} \\
& \times F\left(\beta,\left\{[\varepsilon+\gamma(\varepsilon) / 2]^{2}+\Delta^{2}\right\}^{1 / 2}, \varepsilon+\gamma(\varepsilon) / 2, \gamma(\varepsilon)\right) .
\end{aligned}
$$

The temperature $\beta_{c}$ therefore satisfies the equation

$$
\begin{aligned}
2= & g \rho \int_{-\delta \beta_{c}}^{\delta \beta_{c}} \frac{\mathrm{d} x}{\left|x+(1 / 2) \beta_{\mathrm{c}} \gamma\left(\beta_{\mathrm{c}}^{-1} x\right)\right|} \\
& \times F\left[1,\left|x+(1 / 2) \beta_{\mathrm{c}} \gamma\left(\beta_{\mathrm{c}}^{-1} x\right)\right|, x+(1 / 2) \beta_{\mathrm{c}} \gamma\left(\beta_{\mathrm{c}}^{-1} x\right), \beta_{\mathrm{c}} \gamma\left(\beta_{\mathrm{c}}^{-1} x\right)\right] .
\end{aligned}
$$


If $\delta \beta_{\mathrm{c}} \gg 1$ and the integrand on the rhs of Eq. (34) vanishes sufficiently fast as $x \rightarrow \pm \infty$, then

$$
\begin{aligned}
& \left(\beta_{\mathrm{c}} \delta\right)^{-1} \exp \left(\int_{-\delta \beta_{\mathrm{c}}}^{\delta \beta_{\mathrm{c}}} \frac{\mathrm{d} x^{\prime}}{\left|x^{\prime}+(1 / 2) \beta_{\mathrm{c}} \gamma\left(\beta_{\mathrm{c}}^{-1} x^{\prime}\right)\right|}\right. \\
& \left.\quad \times F\left[1,\left|x^{\prime}+(1 / 2) \beta_{\mathrm{c}} \gamma\left(\beta_{\mathrm{c}}^{-1} x^{\prime}\right)\right|, x^{\prime}+(1 / 2) \beta_{\mathrm{c}} \gamma\left(\beta_{\mathrm{c}}^{-1} x^{\prime}\right), \beta_{\mathrm{c}} \gamma\left(\beta_{\mathrm{c}}^{-1} x^{\prime}\right)\right]\right) \\
& \quad=\lim _{x \rightarrow \infty} x^{-1} \exp \left(\int_{-x}^{x} \frac{\mathrm{d} x^{\prime}}{\left|x^{\prime}+(1 / 2) \beta_{\mathrm{c}} \gamma\left(\beta_{\mathrm{c}}^{-1} x^{\prime}\right)\right|}\right. \\
& \left.\quad \times F\left[1,\left|x^{\prime}+(1 / 2) \beta_{\mathrm{c}} \gamma\left(\beta_{\mathrm{c}}^{-1} x^{\prime}\right)\right|, x^{\prime}+(1 / 2) \beta_{\mathrm{c}} \gamma\left(\beta_{\mathrm{c}}^{-1} x^{\prime}\right), \beta_{\mathrm{c}} \gamma\left(\beta_{\mathrm{c}}^{-1} x^{\prime}\right)\right]\right)=c_{\gamma} .
\end{aligned}
$$

Thus

$$
\frac{2}{g \rho}=\ln \left(\beta_{c} \delta c_{\gamma}\right)
$$

and

$$
\beta_{\mathrm{c}}^{-1}=\delta c_{\gamma} \exp \left(-\frac{2}{g \rho}\right)
$$

$T_{\mathrm{c}}$ can be therefore increased to exceed $T_{c \text { (BCS) }}$ by allowing $|x+\gamma(x) / 2|$, where $\gamma(x)=-2|x|+\sigma(x)$, to assume values sufficiently small on a sufficiently large interval above zero. Similarly, if $\gamma(x)=2|x|+\sigma(x)$ with $|\sigma(x)| \ll|x|$ and $g_{k^{\prime} k^{\prime}}=$ $\left[g_{1} \chi_{-}(k)+g_{2} \chi_{+}(k)\right]\left[g_{1} \chi_{-}\left(k^{\prime}\right)+g_{2} \chi_{+}\left(k^{\prime}\right)\right]$, then $T_{\mathrm{c}}$ can be raised by allowing $|x+\gamma(x) / 2|$ to assume sufficiently small values on a sufficiently large interval below zero.

$$
\begin{aligned}
& \text { If } \gamma(x)=0 \text {, then } T_{\mathrm{c}}<T_{\mathrm{c}(\mathrm{BCS})}, \text { because for such } \gamma \\
& \beta_{\mathrm{c}}{ }^{-1}=c_{0} \delta \exp \left[-(g \rho)^{-1}\right]
\end{aligned}
$$

with

$$
\begin{aligned}
c_{0}= & \lim _{x \rightarrow \infty} x^{-1} \exp \left(\int_{0}^{x} \mathrm{~d} x^{\prime} x^{\prime-1} \frac{\sinh x^{\prime} \cosh x^{\prime}}{\left(\cosh x^{\prime}+1\right)^{2}}\right) \\
& <\lim _{x \rightarrow \infty} x^{-1} \exp \left(\int_{0}^{x} \mathrm{~d} x^{\prime} x^{\prime-1} \tanh \left(x^{\prime} / 2\right)\right)=c_{\mathrm{BCS}}=1.14 .
\end{aligned}
$$

The inequality $T_{\mathrm{c}}<T_{\mathrm{c}(\mathrm{BCS})}$ for $\gamma=0$ shows that interaction $V$ is weaker than $V_{\mathrm{BCS}}$, which agrees with the weakness of the isotope effect in HTSC and confirms the, generally accepted, decisive role of the pair-binding potential $W$ in raising $T_{\mathrm{c}}$ in these materials, e.g. [20-22].

Consider now Eq. (32) for $\mu$ if $g_{k_{k} k^{\prime}}=g \chi(k) \chi\left(k^{\prime}\right)$ for $k \neq k^{\prime}$.In the range of low temperatures (large $\beta$ ) the term $\sum_{\mathbb{k}_{k}} 2 G\left(\beta, E_{\boldsymbol{k}}, \nu_{k}, \gamma_{k}\right)$ on the left hand side of Eq. (32) is negligible compared to the first two summands. The resulting simplified equation is not solvable in general. For $\gamma_{k}=0$ the solution is $\mu=\mu_{\mathrm{F}}$.

Another difference between the thermodynamics of $H$ and $H_{\mathrm{BCS}}$ is in the dependence of the ratio $\Delta(\beta) \Delta(\infty)^{-1}$ on the temperature. In BCS theory this ratio depends exclusively on $T T_{c}^{-1}$. This is not the case in the present model due 
to the fact that $\beta$ enters into $F$ through $\beta E_{k}, \beta \nu_{k}, \beta \gamma_{k}$ and not exclusively through $\beta E_{R}$. We have examined to what extent the plot of $\Delta(\beta) \Delta(\infty)^{-1}$ resulting from Eq. (33) for $\gamma(\varepsilon)=-2|\varepsilon|$ (in which case conditions (B), (C) are fulfilled and the gap in the excitation spectrum equals $\Gamma(\beta)=2 \Delta(\beta)$ ) can be adjusted to fit the experimental data on $\Delta(\beta) \Delta(\infty)^{-1}$ determined on two types of junctions for the BSCCO superconductor and depicted in Refs. [23, 24]. For $\gamma(\beta)=-2|\varepsilon|$, Eq. (9) for $\Delta(\infty)$ takes the form

$$
g \rho=\frac{4}{4 \delta \beta_{\mathrm{c}} a^{-1}+\operatorname{arcsinh}\left(4 \delta \beta_{\mathrm{c}} a^{-1}\right)}
$$

where $a=2 \Delta(\infty) \beta_{\mathrm{c}}$ and Eq. (34) reduces to

$$
\begin{aligned}
2= & g \rho\left(\int_{-\delta \beta_{\mathrm{c}}}^{0} \frac{\sinh (4 x)}{2 x[\cosh (4 x)+4 \exp (x) \cosh (2 x)+2 \exp (2 x)+1)]} \mathrm{d} x\right. \\
& \left.+\int_{0}^{\delta \beta_{c}} \frac{\mathrm{d} x}{[1+\exp (-x)]^{2}}\right) .
\end{aligned}
$$

Given $a$, Eqs. (37), (38) allow us to determine $g \rho$ and $\delta \beta_{\mathrm{c}}$. For the choice $a=2.5$, similar as in other fitting methods (e.g. Ref. [24]), one obtains $\delta \beta_{\mathrm{c}}=7.17$, $g \rho=0.2738$. The resulting graph of $\Delta(\beta) \Delta(\infty)^{-1}$, with $2 \Delta(\infty)=1.81 \times 10^{-2} \mathrm{eV}$ for $T_{\mathrm{c}}=84 \mathrm{~K}$ [24], is depicted in Fig. 1 and compared with experimental data on BSCCO of Ref. [24]. Another comparison with the $\Delta(\beta) \Delta(\infty)^{-1}$ data of Refs. [23, 24] for BSCCO and $T_{\mathrm{c}}=85 \mathrm{~K}$, with $a=2.025, \delta \beta_{\mathrm{c}}=124.783$,

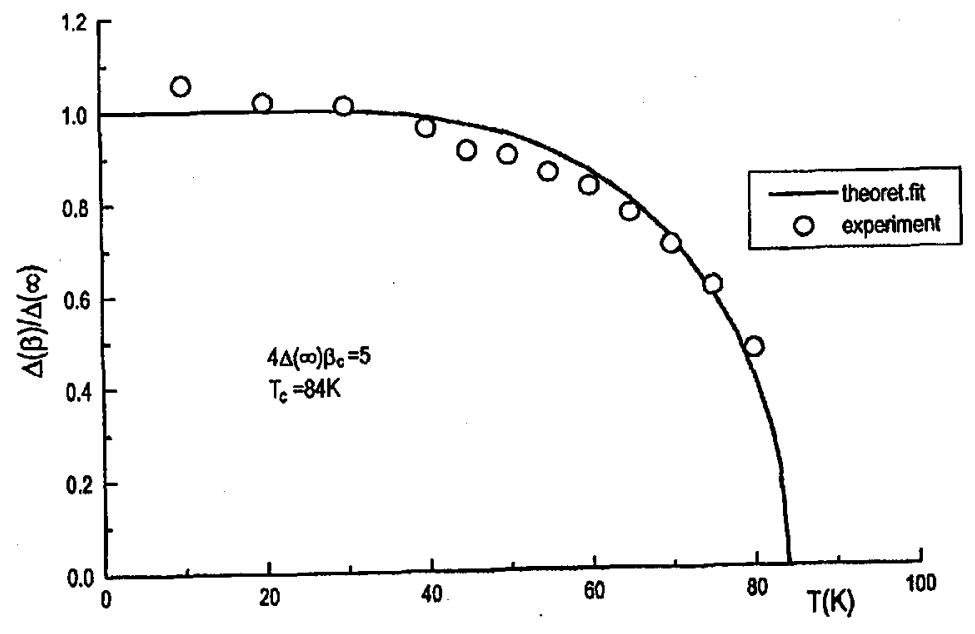

Fig. 1. Temperature dependence of $\Delta(\beta) / \Delta(\infty)$ for a BSCCO/BSCO/Au tunnel junction. The solid line is the $\Delta(\beta) / \Delta(\infty)$ function resulting from Eq. (33) fitted to $a=2.5, T_{c}=84 \mathrm{~K}$. Experimental data (o) after Ref. [24]. 


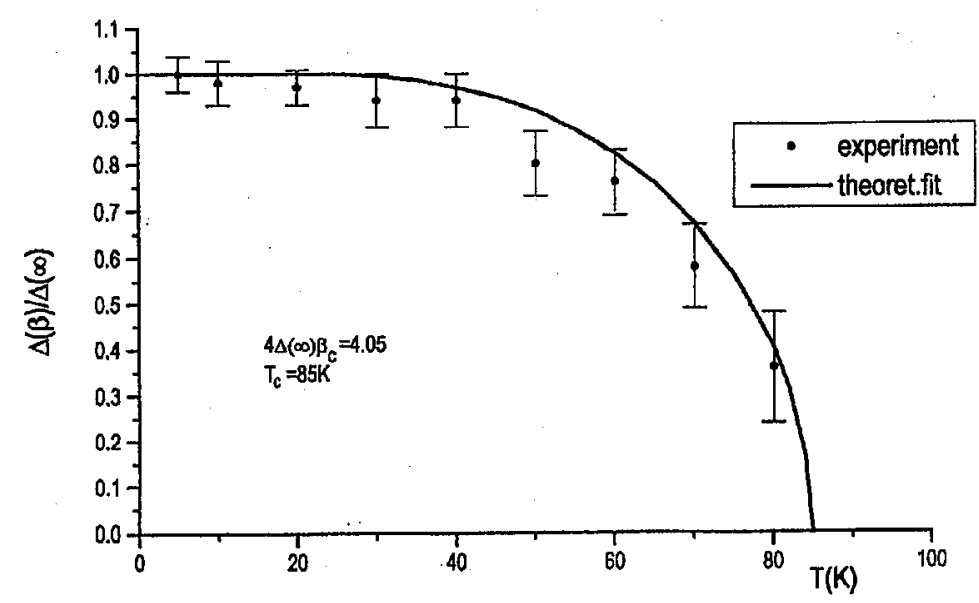

Fig. 2. Temperature dependence of $\Delta(\beta) / \Delta(\infty)$ for a BSCCO/Nb junction. The solid line is the $\Delta(\beta) / \Delta(\infty)$ function resulting from Eq. (33) fitted to $a=2.025$, $T_{c}=85 \mathrm{~K}$. Experimental data $(\bullet)$ after Refs. $[23,24]$.

$g \rho=0.016,2 \Delta(\infty)=1.48 \times 10^{-2} \mathrm{eV}$ is given in Fig. 2. For the crude choice $\gamma(\varepsilon)=-2|\varepsilon|$, agreement with experiment appears to be satisfactory in both cases.

Another confrontation with HTSC experimental data can be obtained by comparing the values of $2 a=2 \Gamma(\infty) / k_{\mathrm{B}} T_{\mathrm{c}}$. In HTSC this ratio assumes values between 2.4 and 11 [24]. In the present model, for $\gamma(\varepsilon)=-2|\varepsilon|, 2 a=5$ and 4.05 in the two cases discussed above. In general, for this choice of $\gamma(\varepsilon), 2 a$ depends on the value of $g \rho$ according to Eq. (37) and

$$
\lim 2 a=0 \text { as } g \rho \rightarrow 0, \quad \lim 2 a=\infty \text { as } g \rho \rightarrow \infty .
$$

Thus, all non-negative values of $2 a$ are admissible and the range [2.4,11] observed in HTSC is covered by the present model. Further properties of the system described by $H$ are under investigation.

\section{Asymptotic exactness of the variational procedure for $H$}

In Ref. [10] Czerwonko developed a method of evaluating the infinite-volume limit of free energy density for a Fermi gas with BCS attraction and repulsion between fermions with equal momenta and opposite spins (described by $W$ with $\gamma_{k}>0$ ). His method is applicable to the Hamiltonian $H$ defined in Eq. (5) with $g_{k k^{\prime}}$ of the form $g_{k k^{\prime}}=\lambda L^{-3} g_{k} g_{k^{\prime}}, L^{3}$ denoting the volume of the system, and allows us to prove the asymptotic equality

$$
\lim _{L \rightarrow \infty}\left(-\beta L^{3}\right)^{-1} \ln \operatorname{Tr} \exp (-\beta H)=\lim _{L \rightarrow \infty} \min _{\rho_{0}} L^{-3} \mathcal{F}\left[\rho_{0}\right]
$$

where $\mathcal{F}\left[\rho_{0}\right]$ is given by the expression $(30)$. In order to carry out this proof, let us first note that the sum of diagonal terms in the potential $V$ with $g_{\boldsymbol{k} \boldsymbol{k}^{\prime}}=\lambda L^{-3} g_{\boldsymbol{k}} g_{\mathbf{k}^{\prime}}$, viz.,

$$
\lambda L^{-3} \sum_{k} g_{k} g_{k} b_{k}^{*} b_{-k}^{*} b_{-k} b_{k}
$$


is uniformly bounded in $L$ if $g_{\boldsymbol{k}}$ is square-integrable on $\mathcal{R}^{3}$ :

$$
L^{-3}\left\|\lambda \sum_{k} g_{k} g_{k} b_{k k}^{*} b_{-k}^{*} b_{-k} b_{k}\right\| \leq|\lambda| L^{-3} \sum_{k} g_{k}^{2} \leq(2 \pi)^{-3}|\lambda| \int_{\mathcal{R}^{3}} g_{\mathbf{k}}^{2} \mathrm{~d}^{3} k
$$

and therefore does not contribute to $L^{-3} \ln \operatorname{Tr} \exp (-\beta H)$ in the limit $L \rightarrow \infty$, $n \rightarrow \infty, n L^{-3}=d$. This form of $g_{k_{k^{\prime}}}$ thus fulfils asymptotically the requirements imposed in the Introduction.

Let us write $H$, with $g_{\mathbf{k} \mathbb{k}^{\prime}}=\lambda L^{-3} g_{\mathbf{k}} g_{k^{\prime}}$, in the form

$$
H=\sum_{\boldsymbol{p} \sigma} \varepsilon_{p} n_{\boldsymbol{p} \sigma}-\lambda L^{-3} B^{*} B+\sum_{\boldsymbol{p}} \gamma_{p} n_{\boldsymbol{p}+} n_{\boldsymbol{p}-},
$$

where $B=\sum_{k} g_{k} b_{-k} b_{k} . H$ can be rewritten as

$$
\begin{gathered}
H=\sum_{\boldsymbol{p} \sigma} \varepsilon_{p} n_{\boldsymbol{p} \sigma}-\lambda L^{-3}\left(B^{*}+B\right)\langle B\rangle+\sum_{\boldsymbol{p}} \gamma_{p} n_{\boldsymbol{p}+} n_{\boldsymbol{p}-}+\lambda L^{-3}\langle B\rangle^{2} \\
-\lambda L^{-3}\left(B^{*}-\langle B\rangle\right)(B-\langle B\rangle) \equiv H^{\prime}-\lambda L^{-3}\left(\Delta B^{*}\right)(\Delta B),
\end{gathered}
$$

where

$$
\left\langle B^{*}\right\rangle=\langle B\rangle=\left[\operatorname{Tr} \exp \left(-\beta H^{\prime}\right)\right]^{-1} \operatorname{Tr}\left[B \exp \left(-\beta H^{\prime}\right)\right],
$$

$\Delta B=B-\langle B\rangle$. The gauge invariance of $H$ admits the choice $\langle B\rangle=\operatorname{Re}\langle B\rangle=\left\langle B^{*}\right\rangle$. The thermodynamical perturbation method $[10,25-29]$ for the statistical sum $Z_{H}=\operatorname{Tr} \exp (-\beta H)$ then yields

$$
Z_{H}=A \operatorname{Tr} \exp \left(-\beta H^{\prime}\right), \quad \text { where } \quad \lim _{L \rightarrow \infty} L^{-3} \ln A=0 .
$$

Thus in order to evaluate $\lim L^{-3} \ln Z_{H}$, as $L \rightarrow \infty$, it suffices to diagonalize $H^{\prime}$. This Hamiltonian can be written as

$$
H^{\prime}=\sum_{\boldsymbol{p}>0} H_{\boldsymbol{p}}+\lambda L^{-3}\langle B\rangle^{2}
$$

with

$$
\begin{aligned}
H_{\boldsymbol{p}}= & \varepsilon_{p} \sum_{\sigma}\left(n_{\boldsymbol{p} \sigma}+n_{-\boldsymbol{p} \sigma}\right)-2 \lambda g_{\boldsymbol{p}} L^{-3}\langle B\rangle\left(b_{\boldsymbol{p}}^{*} b_{-\boldsymbol{p}}^{*}+b_{\boldsymbol{p}} b_{-\boldsymbol{p}}\right) \\
& +\gamma_{p}\left(n_{\boldsymbol{p}+} n_{\boldsymbol{p}-}+n_{-\boldsymbol{p}+} n_{-\boldsymbol{p}-}\right) .
\end{aligned}
$$

$H_{p}$ acts in the 16-dimensional space of states

$$
\left(a_{\mathbf{p}+}^{*}\right)^{n_{1}}\left(a_{\mathbf{p}-}^{*}\right)^{n_{2}}\left(a_{-\boldsymbol{p}+}^{*}\right)^{n_{3}}\left(a_{-p_{-}-}^{*}\right)^{n_{4}}|0\rangle \text {, }
$$

where $n_{i}=0,1, i=1,2,3,4$. All the states (44), except for the two with $n_{1}=n_{2}=$ $n_{3}=n_{4}=0$ and $n_{1}=n_{2}=n_{3}=n_{4}=1$, prove to be eigenstates of $H_{p}$. Due to the commutation relations fulfilled by $H_{\boldsymbol{p}}$, viz.,

$$
\left[H_{p}, 2 S\right]=0, \quad\left[H_{p}, \Lambda_{\alpha}\right]=0,
$$

where

$$
2 S=\sum_{\alpha= \pm 1} \sum_{\sigma= \pm 1} \sigma n_{\alpha p, \sigma}, \quad \Lambda_{\sigma}=n_{p, \sigma}-n_{-p,-\sigma}
$$

denote the spin projection and two seniorities $\Lambda_{+}, \Lambda_{-}$, the diagonalization of $H_{p}$ can be carried out independently in the invariant subspaces of $H_{p}$ with fixed 
eigenvalues of $2 S$ and $\Lambda_{+}, \Lambda_{-}$. This procedure reveals the following eigenstructure of $H_{p}$ :

\begin{tabular}{|c|c|c|c|c|c|}
\hline & Eigenvector & $H_{p}$ & $2 S$ & $\Lambda_{+}$ & $\Lambda_{-}$ \\
\hline 1. & $|1000\rangle$ & $\varepsilon_{p}$ & 1 & 1 & 0 \\
\hline 2. & $|0100\rangle$ & $\varepsilon_{p}$ & -1 & 0 & 1 \\
\hline 3. & $|0010\rangle$ & $\varepsilon_{p}$ & 1 & 0 & -1 \\
\hline 4. & $|0001\rangle$ & $\varepsilon_{p}$ & -1 & -1 & 0 \\
\hline 5. & $|1010\rangle$ & $2 \varepsilon_{p}$ & 2 & 1 & -1 \\
\hline 6. & $|0101\rangle$ & $2 \varepsilon_{p}$ & -2 & -1 & 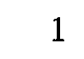 \\
\hline 7. & $|1001\rangle$ & $2 \varepsilon_{p}$ & 0 & 0 & \\
\hline 8. & $|0110\rangle$ & $2 \varepsilon_{p}$ & 0 & 0 & \\
\hline 9. & $|1100\rangle$ & $2 \varepsilon_{p}+\gamma_{p}$ & 0 & 1 & \\
\hline & $|0011\rangle$ & $2 \varepsilon_{p}+\gamma_{p}$ & 0 & -1 & -1 \\
\hline 11. & $|1110\rangle$ & $3 \varepsilon_{p}+\gamma_{p}$ & 1 & 1 & \\
\hline 12 . & $|0111\rangle$ & $3 \varepsilon_{p}+\gamma_{p}$ & -1 & -1 & \\
\hline 13. & $|1101\rangle$ & $3 \varepsilon_{p}+\gamma_{p}$ & -1 & 0 & \\
\hline 14. & $|1011\rangle$ & $3 \varepsilon_{p}+\gamma_{p}$ & 1 & 0 & -1 \\
\hline 15. & $u_{\boldsymbol{p}}|0000\rangle+v_{\boldsymbol{p}}|1111\rangle$ & $2 \varepsilon_{p}+\gamma_{p}-2 E_{p}$ & 0 & 0 & \\
\hline 16. & $u_{p}|1111\rangle-v_{p}|0000\rangle$ & $2 \varepsilon_{p}+\gamma_{p}+2 E_{p}$ & 0 & 0 & \\
\hline
\end{tabular}

where $u_{\boldsymbol{p}}^{2}, v_{\boldsymbol{p}}^{2}$ are given by Eq. (8) with

$$
\Delta_{p}=\lambda L^{-3} g_{p}\langle B\rangle \text {. }
$$

Evaluation of $Z_{H^{\prime}}=\operatorname{Tr} \exp \left(-\beta H^{\prime}\right)$ is now a matter of some simple algebra. One obtains

$$
\begin{aligned}
Z_{H^{\prime}} & =\exp \left(-\lambda \beta L^{-3}\langle B\rangle^{2}\right) \prod_{\boldsymbol{p}>0} 2 \exp \left(-2 \beta \nu_{p}\right) \\
& \times\left[\cosh \left(2 \beta E_{\boldsymbol{p}}\right)+4 \exp \left(\beta \gamma_{p} / 2\right) \cosh \beta \nu_{p}+2 \exp \left(\beta \gamma_{p}\right)+1\right]
\end{aligned}
$$

and Eq. (41) for $\langle B\rangle$, written as

$$
\frac{\partial}{\partial \lambda} \beta^{-1} \ln Z_{H H^{\prime}}=L^{-3}\langle B\rangle^{2}
$$

takes the form

$$
\frac{1}{2} L^{-3} \sum_{p} \lambda g_{p}^{2}\langle B\rangle^{2} E_{p}^{-1} F\left(\beta, E_{p}, \nu_{p}, \gamma_{p}\right)=\langle B\rangle^{2}
$$

and reduces to Eq. (29) with $g_{\boldsymbol{p} \boldsymbol{p}^{\prime}}=\lambda L^{-3} g_{\boldsymbol{p}} g_{\boldsymbol{p}^{\prime}}$, for $\Delta_{\boldsymbol{p}}$ defined by Eq. (46). Using Eq. (48), one finds that the free energy $\mathcal{F}=-\beta^{-1} \ln Z_{H^{\prime}}$ is equal to $\mathcal{F}\left[\rho_{0}\right]$ given by the expression (30). This proves, together with Eq. (42), the equality (39) and establishes asymptotic exactness of the minimization procedure carried out in Secs. 2-4. 


\section{Conclusions}

We have demonstrated that the Fermi gas with the Hamiltonian $H$ is asymptotically solvable in the infinite-volume limit. The ground state (6) is a BCS-type product state of bound quadruples, the excitations are fermions but excitation energies are not simply additive. The system exhibits a 2 nd order phase transition: at $T>T_{\mathrm{c}}$ only free fermions and free bound pairs are present, at $T<T_{\mathrm{c}}$ the system behaves like a gas of fermion quasiparticles with energies which are not additive. A gap is present in the spectrum which depends on the form of the pair-binding potential $W$. The transition temperature $T_{\mathrm{c}}$ also strongly depends on $W$ and exceeds $T_{\mathrm{c}(\mathrm{BCS})}$ if $|\varepsilon+\gamma(\varepsilon) / 2|$ is sufficiently small in a sufficiently large range. The experimentally measured temperature dependences of the gap parameter of the BSCCO superconductor have been compared with the theoretical dependences resulting from $H$ with $W=-\sum_{k} 2\left|\varepsilon_{k}\right| n_{k+} n_{k-}$, adjusted bandwidth $\delta$ and zero-temperature gap parameter $2 \Delta(\infty)=2 a \beta_{c}^{-1}$ for two values of $2 a$, viz., $2 a=5,4.05$. The theoretical curves agree qualitatively with the experimental ones. Further questions relating to the Hamiltonians $H$ and $H^{\prime}$, in particular quantitative agreement with experiment for other choices of $\gamma(\varepsilon)$, are under investigation.

\section{Appendix A}

For the 1-parameter model with $\gamma_{k}=2 \gamma \lambda_{k}, \gamma>-1$, Eq. (10) assumes the form

$$
\sum_{\lambda_{k}<\mu-\delta} 2+\rho \int_{\mu-\delta}^{\mu+\delta}\left(1-\frac{\varepsilon(1+\gamma)-\mu}{\left\{[\varepsilon(1+\gamma)-\mu]^{2}+\Delta^{2}\right\}^{1 / 2}}\right) \mathrm{d} \varepsilon=n
$$

and after performing integration simplifies to

$$
\begin{aligned}
& \sum_{\lambda_{k}<\mu} 2=n+\rho(1+\gamma)^{-1} \\
& \quad \times\left(\left\{[\mu \gamma+\delta(1+\gamma)]^{2}+\Delta^{2}\right\}^{1 / 2}-\left\{[\mu \gamma-\delta(1+\gamma)]^{2}+\Delta^{2}\right\}^{1 / 2}\right)
\end{aligned}
$$

with the solution $\mu<\mu_{\mathrm{F}}$ if $-1<\gamma<0$ and $\mu>\mu_{\mathrm{F}}$ if $\gamma>0$. For $\gamma=0$ the solution is $\mu=\mu_{\mathrm{F}}$.

The gap equation (9), for the same form of $\gamma_{k}$ as assumed above, is

$$
2=g \rho \int_{\mu-\delta}^{\mu+\delta} \mathrm{d} \varepsilon\left\{[\varepsilon(1+\gamma)-\mu]^{2}+\Delta^{2}\right\}^{-1 / 2} .
$$

On performing integration

$$
\int\left(x^{2}+1\right)^{-1 / 2} \mathrm{~d} x=\operatorname{arcsinh} x
$$

and solving for $\Delta$, one obtains the solution (11).

\section{Appendix B}

In order to prove that $x^{-1} F(\beta, x, y, z)$ is decreasing in $x$ for $x>0$, let us first note that $F$ has the form

$$
F(\beta, x, y, z)=\frac{\sinh (2 \beta x)}{\cosh (2 \beta x)+g_{0}(\beta, y, z)}
$$


and therefore, as a function of $x$, has the same growth and convexity properties as $\tanh \beta x$. For any function $F(x)$ with these properties, $\left(x^{-1} F(x)\right)^{\prime}<0$, as can be seen from the Taylor expansion

$$
F(x)=F(0)+x F^{\prime}\left(x^{\prime}\right), \quad x^{\prime} \in(0, x), \quad F(0)=0
$$

and concavity of $F$ for $x>0: F^{\prime}(x)<F^{\prime}\left(x^{\prime}\right)$.

\section{Appendix C}

For $\mathcal{F}\left[\rho_{0}\right]$ given by Eq. $(30)$

$$
\frac{\partial \mathcal{F}}{\partial E_{k:}}=\frac{1}{2}\left[E_{k} \beta \frac{\partial F\left(\beta, E_{k}, \nu_{k}, \gamma_{k}\right)}{\partial \beta E_{k}}-F\left(\beta, E_{\boldsymbol{k}}, \nu_{k}, \gamma_{k}\right)\right]\left(1-\frac{\nu_{k}^{2}}{E_{k}^{2}}\right) \text {. }
$$

Since $\nu_{k}^{2} E_{\boldsymbol{k}}^{-2} \leq 1$, the proof of the inequality

$$
\mathcal{F}\left[\Delta_{\boldsymbol{k}} \neq 0\right]<\mathcal{F}\left[\Delta_{\boldsymbol{k}}=0\right]
$$

can be carried out by demonstrating that

$$
\frac{\partial}{\partial \beta E_{k}}\left(\beta E_{k}\right)^{-1} F\left(\beta, E_{\mathbf{k}}, \nu_{k}, \gamma_{k}\right)<0 .
$$

This has been done in Appendix B.

\section{Acknowledgment}

We would like to thank Prof. J. Czerwonko for his comments and suggestions.

\section{References}

[1] P.W. Anderson, Science 235, 1196 (1987).

[2] P.W. Anderson, G. Baskaran, Z. Zou, T. Hsu, Phys. Rev. Lett. 58, 2790 (1987).

[3] C.P. Enz, Helv. Phys. Acta 61, 741 (1988).

[4] R. Micnas, J. Ranninger, S. Robaszkiewicz, Rev. Mod. Phys. 62, 113 (1990).

[5] High-Temperature Superconductivity, Proc. Los Alamos Symp. 1989, Eds. K.S. Bedell, D. Coffey, D.E. Meltzer, D. Pines, J.R. Schrieffer, Addison-Wesley, Redwood City 1990.

[6] T.M. Rice, K. Ueda, H.R. Ott, H. Rudiger, Phys. Rev. B 31, 594 (1985); J. Spałek, W. Wójcik, Solid State Commun. 60, 119 (1986).

[7] J. Spalek, W. Wójcik, Phys. Rev. B 37, 1532 (1988).

[8] J. Spałek, W. Wójcik, Acta Phys. Pol. A 85, 357 (1994).

[9] J. Czerwonko, Physica $C$ 235-240, 2307 (1994).

[10] J. Czerwonko, Mol. Phys. Rep. 12, 79 (1995).

[11] B. Batlogg, S.W. Cheong, G.A. Thomas, S.L. Cooper, L.W. Rupp Jr., D.H. Rapkine, A.S. Cooper, Physica C 185-189, 1385 (1991).

[12] T.A. Faltens, W.K. Ham, S.W. Keller, K.J. Leary, J.N. Michaels, A.M. Stacy, H.C. zur Loye, D.E. Morris, T.W. Barbee III, L.C. Bourne, M.L. Cohen, S. Hoen, A. Zettl, Phys. Rev. Lett. 59, 915 (1987).

[13] H. Katayama-Yoshida, T. Hirooka, A. Oyamada, Y. Okabe, T. Takahasi, T. Sasaki, A. Ochiai, T. Suzuki, A.J. Mascarenhas, J.I. Pankove, T.F. Ciszek, S.K. Deb, R.B. Goldfarb, Y. Li, Physica C 156, 481 (1988). 
[14] J. Bardeen, L.N. Cooper, J.R. Schrieffer, Phys. Rev. 108, 1175 (1957).

[15] S. Robaszkiewicz, R. Micnas, K.A. Chao, Solid State Commun. 40, 403 (1981).

[16] Ph. Nozieres, S. Schmitt-Rink, J. Low Temp. Phys. 59, 195 (1985).

[17] M. Randeira, J.M. Duan, L.Y. Shieh, Phys. Rev. Lett. 62, 981 (1989).

[18] N.N. Bogolyubov, Nuovo Cimento 7, 794 (1958).

[19] J.G. Valatin, Nuovo Cimento 7, 843 (1958).

[20] J.G. Bednorz, K.A. Müller, Re, . Ylod. Phys. 60, 585 (1988).

[21] J.E. Hirsch, S. Tang, E. Loh, Jr., D.J. Scalpino, Phys. Rev. Lett. 60, 1668 (1988).

[22] B. Batlogg, R.J. Cava, L.W. Rupp, Jr., A.M. Mujsce, J.J. Krajewski, J.P. Remeika, W.F. Peck, Jr., A.S. Cooper, G.P. Espinosa, Phys. Rev. Lett. 61, 1670 (1988).

[23] M. Lee, D.B. Mitzi, A. Kapitulnik, M.R. Beasley, Phys. Rev. B 39, 801 (1989).

[24] Physical Properties of High Temperature Superconductors III, Ed. D.M. Ginsberg, World Scientific, Singapore 1992.

[25] N.N. Bogolyubov, D.N. Zubarev, Yu.A. Tserkovnikov, Dokl. AN SSSR 117, 778 (1957); Zh. Eksp. Teor. Fiz. 39, 120 (1960).

[26] N.N. Bogolyubov, Dubna Preprint P-551 (1960); Selected Papers, Vol. 3, Naukova Dumka, Kiev 1971, p. 110.

[27] J. Czerwonko, Bull. Acad. Pol. Sci. Cl. III 3, 99 (1961); Yadernaya Fizika 2, 14 (1965).

[28] N.N. Bogolyubov, Jr., A Method for Studying Model Hamiltonians, Pergamon, Oxford 1972.

[29] F.W. Nijhoff, H.W. Capel, Physica A 106, 369 (1981). 